\title{
Correction to: A Glycine soja group S2 bZIP transcription factor GsbZIP67 conferred bicarbonate alkaline tolerance in Medicago
} sativa

Shengyang $\mathrm{Wu}^{1,2+}$, Pinghui Zhu ${ }^{1 \dagger}$, Bowei $\mathrm{Jia}^{2}$, Junkai Yang ${ }^{2}$, Yang Shen², Xiaoxi Cai ${ }^{2}$, Xiaoli Sun², Yanming Zhu $u^{1,2^{*}}$ and Mingzhe Sun ${ }^{1,2^{*}}$

\section{Correction to: BMC Plant Biology}

https://doi.org/10.1186/s12870-018-1466-3

Following publication of the original article [1], the author reported that their given name was misspelled. The details are as follows:

Incorrect name in the original article:

Pinhui Zhu

\section{Correct name:}

Pinghui Zhu

The original article has been corrected.

\section{Author details}

${ }^{1}$ Plant Bioengineering Laboratory, Northeast Agricultural University, Harbin 150030, People's Republic of China. ${ }^{2}$ Crop Stress Molecular Biology

Laboratory, Heilongjiang Bayi Agricultural University, Daqing 163319, People's

Republic of China.

Published online: 14 January 2019

\section{Reference}

1. Wu S, et al. A Glycine soja group S2 bZIP transcription factor GsbZIP67

conferred bicarbonate alkaline tolerance in Medicago sativa. BMC Plant Biol. 2018;18:234. https://doi.org/10.1186/s12870-018-1466-3.

\footnotetext{
* Correspondence: ymzhu@neau.edu.cn; kaik127@163.com

† Shengyang Wu and Pinghui Zhu contributed equally to this work.

'Plant Bioengineering Laboratory, Northeast Agricultural University, Harbin

150030, People's Republic of China
} 